\title{
Actuator Array for Use in Minimally Invasive Surgery
}

\author{
H. Fischer, R. Trapp, L. Schüle and B. Hoffmann* \\ Forschungzentrum Karlsruhe GmbH, Hauptabteilung Ingenieurtechnik, Postfach 3640, 76021 Karlsruhe, \\ Germany \\ * Universität Karlsruhe, Institut für Technologie der Elektrotechnik, Hertzstr. 16, 76187. Karlsruhe, \\ Germany
}

\begin{abstract}
For tactile-haptical feedback in minimally invasive surgery, a tactile actuator array consisting of $8 \times 8$ actuators is being built. The system is combined with a tactile optical sensor system mounted in a laparoscopic forceps, providing $8 \times 8$ signals. These signals have to be shown as a true-scale representation through the actuator array. Therefore each measured pressure signal is coded into a corresponding actuating displacement. Each actuator is made out of a SMA-spring conditioned with the one way effect. The SMA spring works against a bias spring and both are mounted on an electrically isolated base pin. The bias spring produces a linear rising force against the SMA-spring for resetting the actuator during the passive cooling time. The whole actuator has a diameter of $1.6 \mathrm{~mm}$, a length of $25 \mathrm{~mm}$ and is mounted at a distance of $3.2 \mathrm{~mm}$ from the next actuator. The resulting force of a single actuator while pressing against the surgeon's fingertip is over $2.5 \mathrm{~N}$, and the actuating displacement is about $3.5 \mathrm{~mm}$. Each pin has analogue adjustment and can be stopped at all positions within these $3.5 \mathrm{~mm}$ deflection. The resetting time is about 10 seconds and is reached with 3 fans mounted on three sides of the actuator array. The aim is to build a minimally invasive palpation system so that the surgeon can 'feel' with his laparoscopic forceps.
\end{abstract}

\section{INTRODUCTION}

In minimally invasive surgery the surgeon reaches the organs inside the body via natural and artificial ports. When instruments are used (e.g. endoscopic forceps), which until now have been associated with high friction, the surgeon's sense of touch is almost completely lost. Also gripping with such forceps stimulates the proprioceptors in the joints of the fingers and arms whereas palpation stimulates the mechanosensors in the fingertips [1]. For this reason, a tactile pressure sensor consisting of $8 \times 8$ measure points was built. The sensor is measuring the pressure distribution between the jaws of a forceps [2]. The resulting pressure distribution on a $0.64 \mathrm{~cm}^{2}$ surface area has to be relayed back onto the fingertips of the surgeon. Therefore, a tactile-haptical actuator array also consisting of $8 \times 8$ actuators was built. Each tactile point in this case corresponds to an actuator, and the magnitude of the pressure force is attributed to the corresponding deflection of the actuator. Each actuator is made of a SMA spring working against a bias spring to reset the actuator during the cooling period [3].

\section{SYSTEM CONFIGURATION}

An actuator pin consists of a SMA-spring which, together with a bias counterspring, is fixed on a ceramic pin. The two springs are separated from each other by a disk which transmits the force. The ceramic pin is $d_{c}=0.8 \mathrm{~mm}$ in diameter, the wire diameter of the SMA-spring is $d_{s}=0.3 \mathrm{~mm}$ and the external diameter of the spring is $d_{e}=1.6 \mathrm{~mm}$. The contact with the spring is effected through laser welding using a silver coated copper-stranded wire. For linear positioning of the actuators, the SMA spring is overdimensioned. This provides for a linear relationship between the deflection and the actuating parameter within the required travel of 
$3.5 \mathrm{~mm}$. The actuator laid out in this way develops a spring force $F_{\max } \approx 2.5 \mathrm{~N}$ when it has reached its maximum actuating travel $\mathrm{s}_{\max }=3.5 \mathrm{~mm}$. The wire diameter of the bias counterspring is $\mathrm{d}_{\mathrm{cs}}=0.25 \mathrm{~mm}$ and the spring travel is $\mathrm{s}_{\mathrm{t}}=4.32 \mathrm{~mm}$. The layout of the actuator pin is shown in Fig. 1a; the actuator array is represented in Fig. Ib.

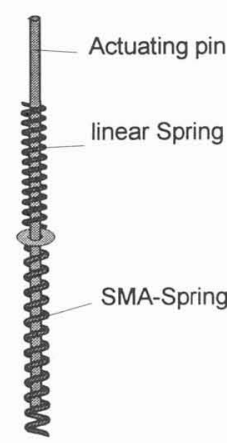

a) Actuator Pin

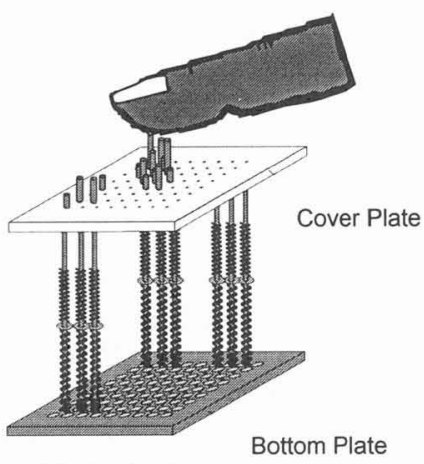

b) Actuator Array

Fig. 1: Actuator pin and actuator array

Cooling the actuator array was provided by three fans. Two of them were attached to the side and one was located underneath the array (not indicated in Fig. 1).

\section{RESULTS}

The electric power introduced into the SMA-spring describes the actuator behaviour [4]. The supplied power is a measure of spring heating and hence of the degree of phase transformation and therefore the deflection. First, the characteristic curves of several actuators were plotted. Next, the thermal interaction among actuators in an array was investigated. Finally, a pressure profile recorded by the sensor in the laparoscopic forceps was represented by the actuators.

\subsection{Characteristic curve of several actuators}

Fig. 2 shows the characteristic curve of deflection as a function of the electric control voltage supplied. The voltage and the current across the SMA-spring correspond to the electric power input and, hence, to the phase transformation. All actuators exhibit approximately the same behaviour. The differences in the characteristic curves are filed in a correction matrix. These characteristic curves are then taken into account when actuators are triggered. 


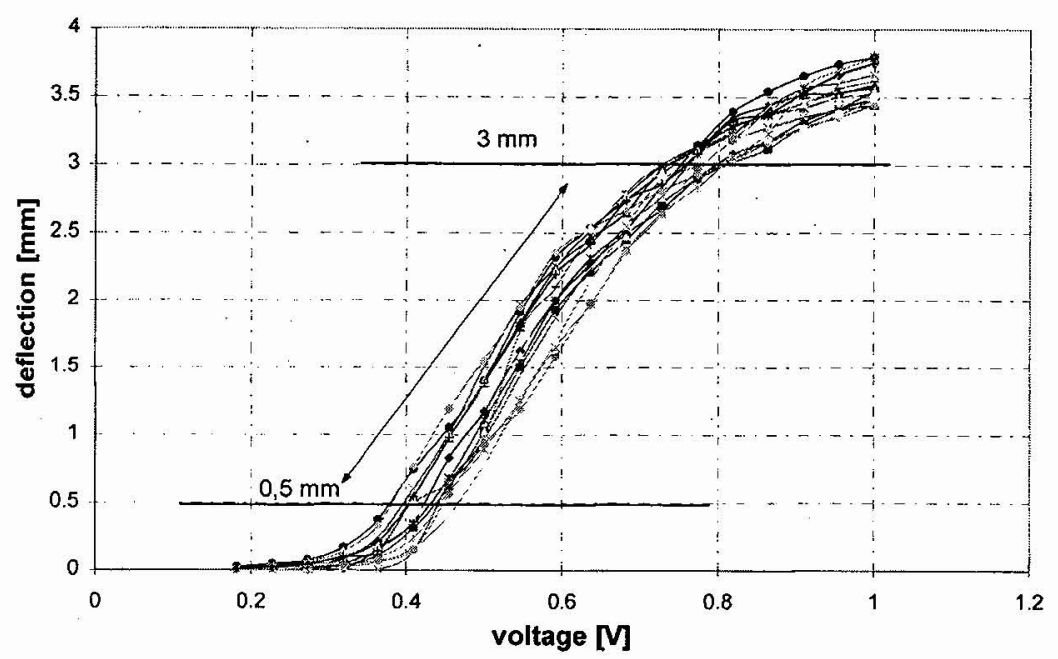

Fig. 2: Characteristic curve of voltage-induced deflection of several actuators

\subsection{Thermal interaction among actuators in the matrix}

Without any cooling system, after a finite period of time, and as a function of the number of triggered pins, all SMA-springs heat to the same temperature, and thus all have the same deflection. Actuators are influenced thermally by directly adjacent pins, as the distance to these neighboring pins is a minimum, and the temperature gradient is a maximum. The pins surrounding the center pin were placed at different levels, and the resulting deflection of the center pin was measured after 30 seconds. These 30 seconds roughly correspond to the time required by the scanner (surgeon's fingertip) to scan the level profile represented. Subsequently, the center pin was set to the different deflection levels, and the surrounding actuators were also deflected at steps of $0.5 \mathrm{~mm}$. This measurement had maximum cooling, that means all fans were in operation.

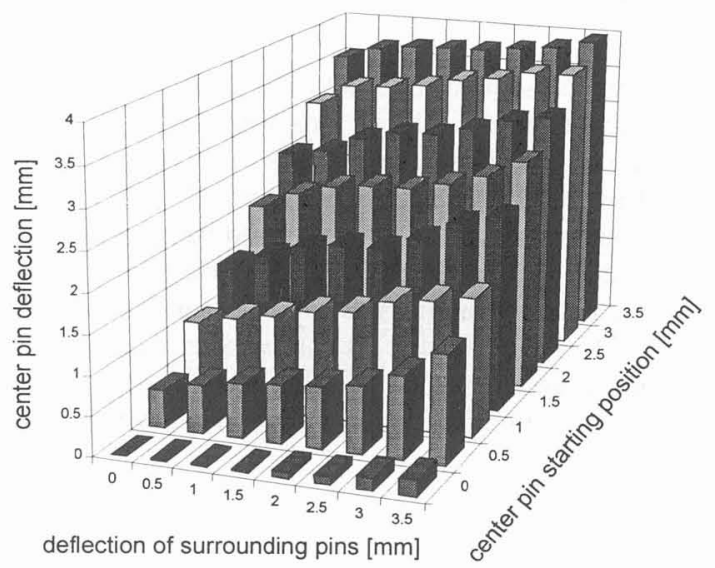

Fig. 3: Thermal influence on the center pin when the surrounding pins are set 
Fig. 3 describes the thermal influence on the center pin while setting the surrounding pins. The surrounding pins were all set to the same deflection. Consequently, in the Z-direction, the change in the center pin can be read from its starting position. If the center pin is not triggered, and if all surrounding pins are deflected to $3.5 \mathrm{~mm}$, the center pin is deflected to $0.203 \mathrm{~mm}$ by the ambient temperature (Fig. 3). If i. e. the center pin has already been deflected to $1.5 \mathrm{~mm}$, it is additionally deflected to $2.483 \mathrm{~mm}$ by the positioning of the surrounding pins. As can be seen from Fig. 3, convection has an additive impact on deflection. So, if the center pin is not triggered, the influence of convection is minimal as the austenite starting temperature $A_{s}$ must be reached first. In order to deflect the center pin, which was not triggered, it must be heated to a temperature above $47^{\circ} \mathrm{C}$. On the other hand, if the pin is triggered to a certain deflection, a slight increase in temperature is sufficient to deflect the pin even further. If the deflections of the surrounding pins are taken into account in triggering a pin, the additional heat input by convection can be balanced out.

\subsection{Pulsed current triggering}

The characteristic curves determined so far were recorded by DC triggering. This ensures setting times of up to 30 seconds (with maximum cooling). However, permanent cooling required for setting the pins needs powerful fans. As, in addition, setting times in excess of 30 seconds are to be made possible, triggering by current pulses has been developed. This helps to reduce the total power requirement for setting the actuators. In this kind of triggering, the increase in ambient temperature of the individual pins is taken into account. As a result of the elevated ambient temperature, less electric power is required for the same deflection. The heating and cooling intervals are shown in Fig. 4. The first heating period takes 22 seconds.

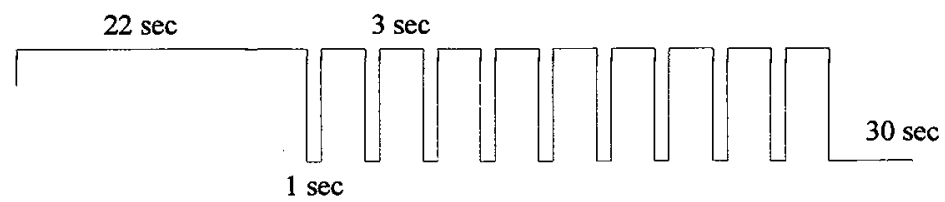

Fig. 4: Development of trigger voltage

In order to reduce the subsequent interaction among pins, the current was turned off for one second and, at the same time, the three fans were started up, removing the heated air. Then, heating was resumed for another three seconds. This cooling and heating cycle was performed nine times, thus allowing the pattern to be scanned over the period of one minute. A wave made up of $2 \times 4$ actuators served as the pattern to be represented. Fig. 5 shows the deflections of the pins of the second line after 22,30, and 60 seconds. Actuator 1 is to travel over a defiection of $1 \mathrm{~mm}$, actuator number 2 is to cover $2 \mathrm{~mm}$, actuator number $3,3 \mathrm{~mm}$, and actuator number 4 is to cover $1.5 \mathrm{~mm}$. It can be seen that the desired deflection is reached by almost all actuators after 22 seconds. After 30 seconds, the actuators have attained their desired deflection. This deflection can be maintained over a period of up to 60 seconds. 


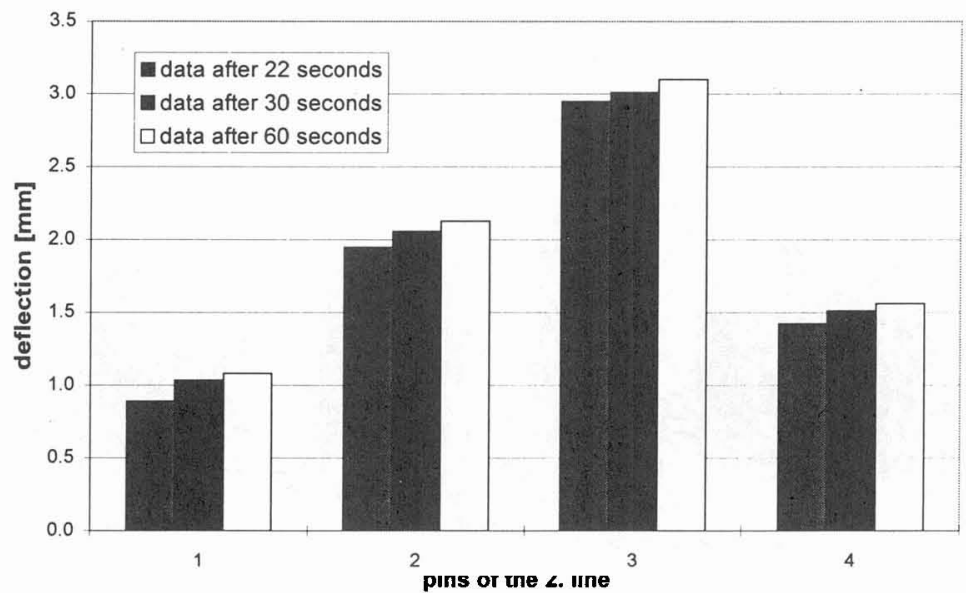

Fig. 5: Deflection of pins at various points in time

Pulsed current modulation is an appropriate way of triggering actuators. Pressure profiles can be scanned in a stable mode over a period of 60 seconds and more. Consequently, this type of triggering is to be used to trigger actuators with the real pressure signals of the optical pressure sensor.

\subsection{Description of sensor signals by the actuator matrix}

The representation of a pressure profile as recorded by the sensor in the endoscopic forceps will be described by the example of a lymphatic node in the pelvic region of a pig. The pressure data correspond to real measurements in an animal experiment. Fig. 6 shows the lymphatic node on the sensor surface.

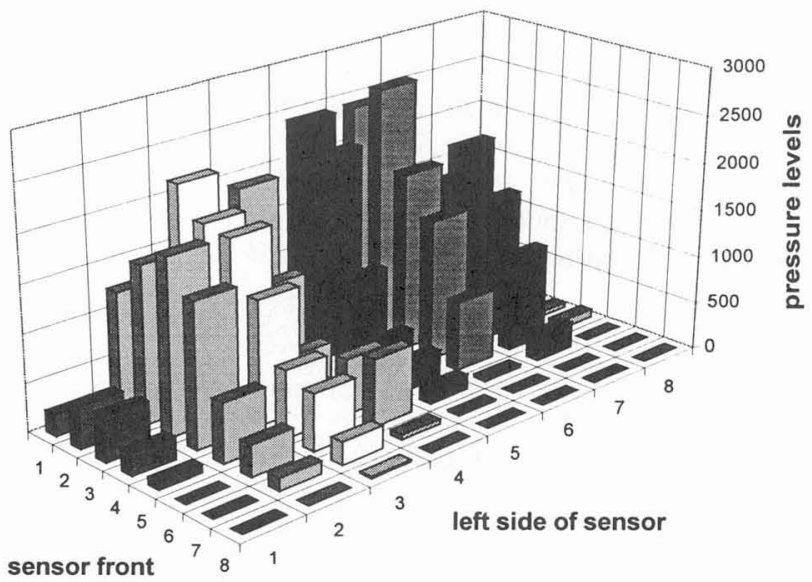

Fig. 6: Sensor signals from the lymphatic node 
The set point values and the actual values of the displacements of the actuators are shown in Fig. 7. The eccentric position of the lymphatic node can be simulated by the actuators.
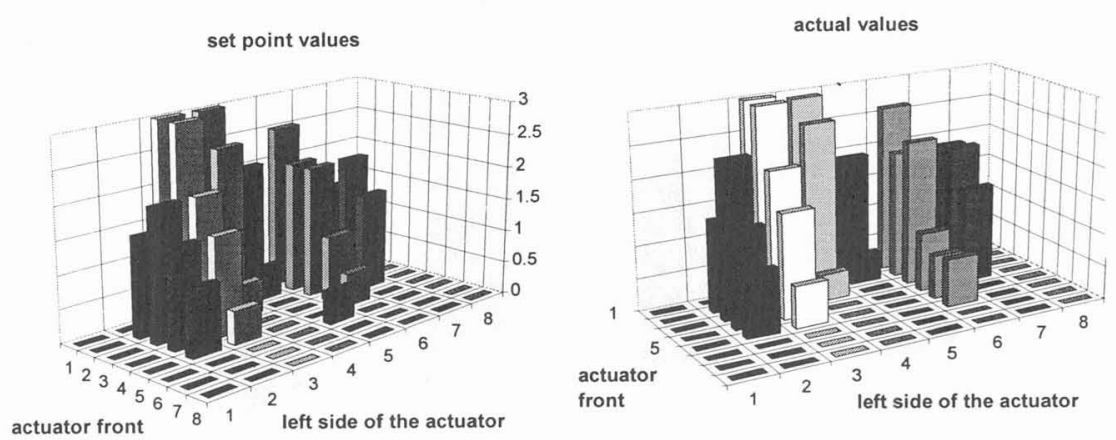

Fig. 7: Bar diagrams of actuators, set point values and actual values after processing by the correction matrix

The forces developed in this process by the actuators are so large that the counterpressure of the finger does not change the position of the actuators. In this way, pressure distributions measured by a tactile sensor in the endoscopic forceps can be represented by actuators and, therefore they can be felt by the surgeon's fingers.

\section{CONCLUSION AND OUTLOOK}

Previous experiments have shown that any linear positioning of the actuators is feasible, provided that the generated heat is adequately removed. In such a case, the actuators can be controlled by the supplied power (by measuring the applied voltage and current). The testing of various cooling techniques is geared to ensuring, constant ambient conditions of the actuators, so that an accuracy of $0.1 \mathrm{~mm}$ of the positioning travel is to be achieved. Pressure distributions of the kind measured by the tactile optical sensor in the endoscopic forceps thus cannot only be represented to the surgeon in a graphical mode, but it is now possible for the first time to allow the surgeon's fingers to feel the pressure distribution. The pulsed current trigger mode developed in combination with the cooling fans can ensure holding times of the pressure profiles of $>60$ seconds. Thermal interaction among the actuators in the dense matrix can thus be reduced considerably.

Attempts are currently under way at FZK to develop a larger number of actuators for a graphic output unit for blind computer users. This would enable blind operators to feel threedimensional graphic representations.

\section{References}

[1] Schmidt R., Thews G.: Physiologie des Menschen, Springer Verlag, 26. Auflage, 1995

[2] Fischer H., Trapp R.: Tactile optical Sensor for Use in minimally invasive Surgery, IOS Press Ohmsma, Amsterdam, 1996

[3] Stöckel D., Hornbogen E., et al.: Legierungen mit Formgedächtnis, Expert Verlag, 1988

[4] Duerig T., Melton K., et al.: Engineering Aspects of Shape, Memory Alloys, ButterworthHeinemann Ltd., London, 1990 